\title{
A New Contourlet-based Compression and Speckle Reduction Method for Medical Ultrasound Images
}

\author{
SeyyedHadi Hashemi-Berenjabad \\ Tarbiat Modares university \\ Electrical and Computer Engineering Department \\ Tehran- Iran
}

\author{
Ali Mahloojifar \\ Tarbiat Modares university \\ Electrical and Computer Engineering Department \\ Tehran- Iran
}

\begin{abstract}
In this paper a threshold based method for speckle reduction and image compression of medical ultrasound images was presented. First, two ultrasound medical image despeckling methods were compared: wavelet-based and contourlet-besd, to find the best. Different measures were used for performance comparison and these methods were implemented both on synthesized data and real ultrasound images. It is found that, performance of the both techniques vary with the level of the speckle noise, and in the case of preserving image details and edges which is very important for medical image processing, contourlet-based method shows better performance over wavelet-based speckle reduction specially in high levels of noise. Then, a new contourlet-based lossy image compression method was presented for medical ultrasound images. In this algorithm, contourlet transform was used for image decomposition. Then, a new thresholding process was applied on the coefficients before quantization. The compression threshold was elected due to coefficients occurrence in the contourlet domain. This algorithm has the ability of simultaneous speckle reduction using another thresholding. Due to this time saving ability, the algorithm can be used in online image transmission systems. The proposed method was implemented on a real ultrasound images and ultrasound phantom image. Results proved that our proposed method has acceptable and good performance over common compression methods such as wavelet-based SPIHT in the case of PSNR.
\end{abstract}

\section{General Terms}

Image Processing, Medical Image Processing

\section{Keywords}

Contourlet Transform, Compression Ratio, Image Compression, Speckle Reduction, PSNR, Ultrasound image, Wavelet

\section{INTRODUCTION}

Among different imaging modalities, ultrasound imaging due to being non-invasive, harmless and cost effective is a popular imaging method. However, ultrasound images suffer from low image quality that is the result of some physical and natural factors such as speckle noise. Despite advantages of digital image technology, digital images are large in size and need large number of bits for storage. The other limitation of these large images is bandwidth that is needed for transmission. So, compression of images was introduced as a solution to overcome these limitations. As a result, in past few decades considerable efforts in the field of medical ultrasound image processing have been directed at development of speckle reduction techniques and image compression methods.
Speckle noise is a phenomenon that accompanies all coherent imaging modalities in which images are produced by interfering echoes of a transmitted waveform that emanate from heterogeneities of the studied objects. The superposition of acoustic echoes coming with random phases and amplitudes tends to produce an intricate interface pattern, known as speckle. Speckle noise obscures and masks diagnostically important details, thereby distracting the diagnosis.

The statistics of the speckle, which generally depend on the microstructure of tissue parenchyma, can be useful for differentiating between either different tissue compositions or types [1]. But there is no consensus on a unified way to interpret and use this useful information.

Due to the importance of the enhancing the quality of ultrasound images, lots of despeckling methods were proposed in order to reduce the speckle noise. Speckle reducing filters originated from the synthetic aperture radar (SAR) community [3]. These filters have been applied to ultrasound images. Filters used in both SAR and ultrasound imaging include: Lee [4], Kuan [5] and Frost [6]. Other methods include Wiener filtering [7], Median filtering [8], Gamma filters [9], anisotropic diffusion [10], wavelet denoising [11], [12], [13], [14]. According to the results, wavelet noise reduction method has acceptable performance [13], [14]. A new image representation was proposed by Minh N. Do is contourlet transform. Contourlet is powerful tool for representing 2D signals and images with soft contours and used for denoising the images [15], [16].

Image compression is a part of data compression which its aim is to reduce the data required to represent the image information. Data is different from information as data are means by which information is transformed. So various amount of data can be used to represent the same amount of information [18].

Data compression is typically characterized into two groups of algorithms: lossless compression and lossy compression. In lossless compression the original image can be reconstructed exactly without any loss, hence it is a reversible process. RunLength Encoding (RLE) is example of lossless compression. Lossless methods suffer from low compression ratios. In lossy techniques, there is some information loss. Lossy compression algorithms have high compression ratios with some errors. These algorithms try to remove unimportant information from images. This information usually is not detectable by human visual system. In the case of medical images lossless methods are preferable but because of storage limitations lossy techniques are used. Due to the importance of image compression and image quality, different standards are introduced in this field. JPEG, JPEG 2000 and DICOM 
are examples of image compression standards. DICOM is a comprehensive set of standards for handling, storing and transmitting information in medical imaging systems such as PACS (Picture Archiving and Communication Systems).

Different image compression methods are presented in literature. Transform based image compression algorithms are very popular in medical community. Discrete Cosine Transform (DCT) and Discrete Wavelet Transform (DWT) are used broadly in this field. DCT based JPEG standard, divides the input image into blocks, DCT is performed on each block then the resulting coefficients are quantized and coded. The main problem of this compression method is blocking artifact [19]. After the introduction of wavelet transform, it has gained broad popularity due to its unique decorrelation property. The most important advantage of DWT based image compression techniques is lack of blocking artifact [20]. After the introduction of DWT, many compression algorithms were proposed to code wavelet coefficient. Among these algorithms, Embedded Zero trees Wavelet (EZW) [22], Set Partitioning in Hierarchical Trees (SPIHT) [5], Embedded Block Coding with Optimized Truncation (EBCOT) [23] are the most popular procedures. Fractal compression algorithm is another compression technique which involves pixel to pixel comparison between the image blocks [24].

This paper is organized as follow. After a brief introduction on image compression and ultrasound despeckling, we describe speckle noise model. In section 3 lossy compression methods is introduced and then, the contourlet transform as a new processing tool is described. In section 5 the proposed method is explained and in part 6 the results are presented and in the last section conclusion on the results and comparison are presented.

\section{SPECKLE NOISE MODEL}

The most important part of recovering a noisy signal is defining its noisy model. In ultrasound imaging there is a general model for speckled images used for filtering and processing goals. This model proposed in [17] is as below:

$$
g(n, m)=f(n, m) u(n, m)+\xi(n, m)
$$

where g, f, $\mathrm{u}$ and $\xi$ stand for the observed envelope image, original image, multiplicative and additive components of the speckle noise, respectively. Here the indices $\mathrm{n}$ and $\mathrm{m}$ denote the axial and lateral indices of the image samples. Moreover evidence exists that the additive component is negligible and we can change (1) into:

$$
g(n, m) \square f(n, m) u(n, m)
$$

If we apply logarithmic transform to both parts of (2), the multiplicative noise is converted to additive noise as follows:

$$
g_{l}(n, m)=f_{l}(n, m)+u_{l}(n, m)
$$

At this stage, the problem of despeckling is reduced to the problem of rejecting the additive noise, and different noise reduction methods could be applied. In this case, the despeckling methods assume the noise to be WGN

\section{LOSSY COMPRESSION}

In lossy image compression methods, image is decomposed using different kinds of transforms. Wavelet and Discrete Cosine Transform are used for transform images. The goal of transformation is to represent the original image in a more effective way. The second process is quantization which is the key issue for lossy methods and it is the difference between lossless and lossy methods. Quantization reduces the symbols used to represent the image. Two types of quantization are used in lossy compression methods: scalar quantization and vector quantization. In scalar quantization each symbol quantized separately but in vector quantization some number of successive symbols form a vector and that vector is quantized. Last part of lossy compression process is entropy encoding. Quantized symbols are encoded using different entropy coding algorithms, like Huffman encoding. Huffman coding is an entropy encoding algorithm used for lossless data compression. In this coding a variable-length code table is used for encoding a source symbol where the variable-length code table has been derived in a particular way based on the estimated probability of occurrence for each possible value of the source symbol. The output of this process is a compressed image bit stream [18]. Fig.1 shows lossy image compression process.

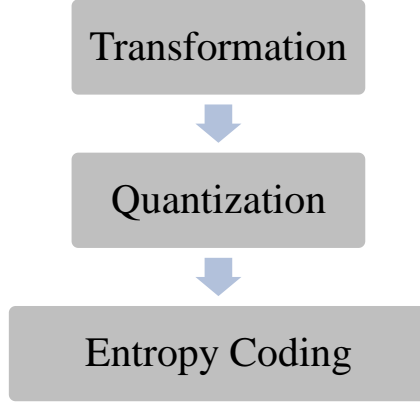

Fig 1: Lossy image compression schematic

\section{CONTOURLET TRANSFORM}

The contourlet transform is a directional transform, capable of capturing contours and fine details in images. The contourlet expansion is composed of basis function oriented at various directions in multiple scales, with flexible aspect ratios. Using this rich set of basic functions, the contourlet transform effectively capture the smooth contours that are dominant feature in images [15]. Contourlets not only possess the main features of wavelets (i.e. multi-scale and time-frequency localization), but also offer a high degree of directionality and anisotropy.

Similar to wavelet, contourlet can decompose the image into different scales. But unlike the wavelet which can only decompose each scale into two directions, contourlet can decompose each scale into any arbitrarily power of two's number of directions and different scales can be decomposed into different number of directions. So, the contourlet uses a double filter-bank structure, namely pyramidal directional filter banks (PDFB) which is composed of Laplacian Pyramid (LP) and Directional Filter Banks (DFB). LP implements multiresolution decomposition to generate multiscale representation of the image. PDFB uses DFB to process subband images from LP and reveal directional details in each scale level. This two stage filter bank is illustrated in figure 2 [15].

Efficiency of contourlet representation of image has been shown for different image processing tasks such as image denoising and compression. Using directional filterbank make it possible to capture important details of the image. In this paper we use contorlet transform as the main tool for image compression. 


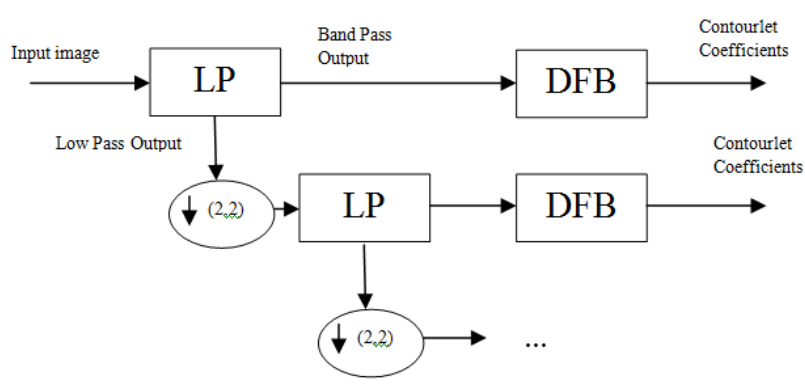

Fig 2: contourlet filter banks

\section{PROPOSED METHOD}

In this section the proposed method for compression and speckle reduction of medical ultrasound images using contourlet transform is presented. First, speckle reduction using contourlet transform was described. Then, the proposed method was described.

\subsection{Speckle Reduction Using Contourlet Transform}

Efficiency of contourlet representation of image has been shown for different image processing tasks such as image denoising and compression. In image denoising, random noise will generate significant wavelet coefficients just like true edges, but is less likely to generate significant contourlet coefficients. So, simple thresholding is efficient in rejecting the speckle noise and separating noise from the texture of the image [16].

The threshold for contourlet denoising is calculated with respect to the variance of speckle noise, as follow:

$$
\lambda=c \sigma^{2}
$$

where $c$ is predefined value and $\sigma^{2}$ is variance of the speckle noise.

Please use a 9-point Times Roman font, or other Roman font with serifs, as close as possible in appearance to Times

\subsection{Contourlet-based Compression and Speckle Reduction}

A new contourlet based image compression method was presented. Contourlet transform used to decompose the image into coefficients. After decomposition, a threshold was selected from contourlet coefficients. Then, a thresholding process was applied on the coefficients and then, a routine quantization process was implemented. In this algorithm choosing the threshold plays a significant role. So, in order to minimize the important information loss, the most frequently occurring contourlet coefficient of the image was selected as the threshold. After thresholding the coefficients is quantized. Huffman coding is applied on quantized coefficients and the coded bit-stream is generated. Block diagram of this algorithm is shown in fig. 3. This threshold based process reduces the number of coefficients necessary to reconstruct the image. Scalar quantization is selected due to its simplicity and performance. The coded bit-stream includes coefficients and contourlet filter information.

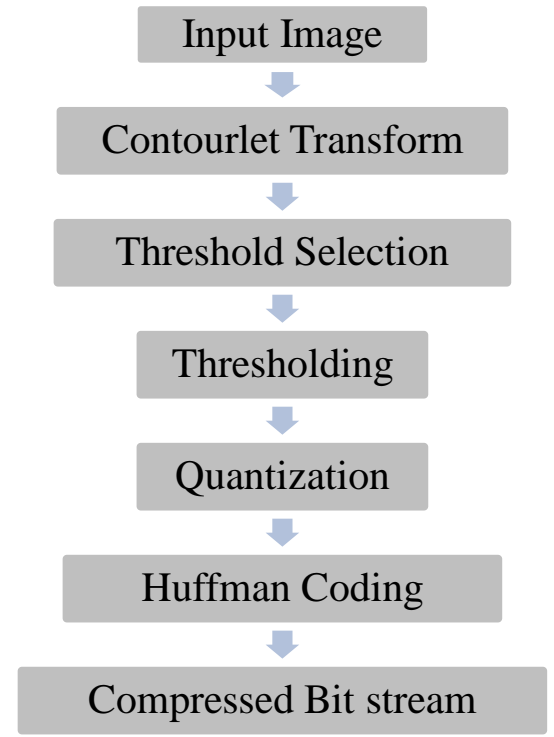

Fig 3: Proposed compression method block diagram

Two thresholds can be used in thresholding stage, one for compression and another for speckle reduction as illustrated in section 5.1. Ultrasound images suffer from the presence of speckle noise. Other methods need another pre-processing step to reduce speckle noise of the image, but our method can reduce speckle during compression process. This property makes this algorithm suitable to use in online medical image transmission systems.

\section{RESULTS}

This paper aims to introduce a contourlet-based technique for image compression and speckle reduction of medical ultrasound images..

\subsection{Performance evaluation of despeckling methods}

In this paper, two speckle reduction methods was implemented and their performance was compared using three important metrics. First, a synthesized ultrasound image having different levels of added speckle noise was used. The aim of this process is to determine the relationship between noise level and method performance. The results of this process are presented in table 1 and figure 4 and 6 , and we notice some changes in measures as the sigma (standard deviation of the speckle noise) grow.

Then, a sample real speckled fetal ultrasound image was used to compare the methods performance on a real data and the results are presented in table 2 and figure 5. In contourlet denoising we use threshold $=3$ due to results obtained in [16] For speckle reduction using wavelet, the soft-thresholding function was used for applying to wavelet coefficient and we use the standard deviation of the HH subband for determining the threshold value. Measures that were used in evaluating the performance of despeckling techniques are PSNR, SNR and FOM that are defined next.

First measure that was used for measuring methods ability in preserving edges and sharp details is Figure of Merit (FOM) which is calculated as follow: 
$F O M=E\left\{\frac{\left\langle\Delta I_{\text {org }}, \Delta I_{\text {est }}\right\rangle}{\left\|I_{\text {org }}\right\|_{F}\left\|I_{\text {est }}\right\|_{F}}\right\}$

In which $\langle\mathrm{a}, \mathrm{b}\rangle$ denotes the standard inner product of $\mathrm{a}$ and $\mathrm{b}$, $\Delta I_{\text {org }}$ and $\Delta I_{\text {est }}$ are the approximation of the original image and denoised image with Laplacian, respectively. The best value of FOM for edge preserving is 1 .

Peak Signal to Noise Ratio (PSNR) is an assessment parameter to measure the performance of the speckle noise removal method. The formula is:

$$
P S N R=10 \log _{10}\left(255^{\wedge} 2 / M S E\right)
$$

where MSE is the Mean Square Error and is used to obtain the total amount of difference between two images. It indicates the average difference of the pixels throughout the image with speckle noise. A lower MSE indicates a smaller difference between the original image and de-noised image. MSE is defined as below:

$M S E=\left(\sum I(x, y)-D I(x, y)\right)^{\wedge} 2 /(m * n)$

In which I is original image with speckle and DI is the denoised image.

Signal to Noise Ratio (SNR) is the last measure used to quantify how much the image is corrupted by noise. Its formula is as follow:

$S N R=10 \log \left(\frac{\max (I)-\min (I)}{\sigma}\right)$

Where I is the image and $\sigma$ is the standard deviation of the image and $\max (\mathrm{I})$ and $\min (\mathrm{I})$ are the maximum and minimum pixel value in the image, respectively.

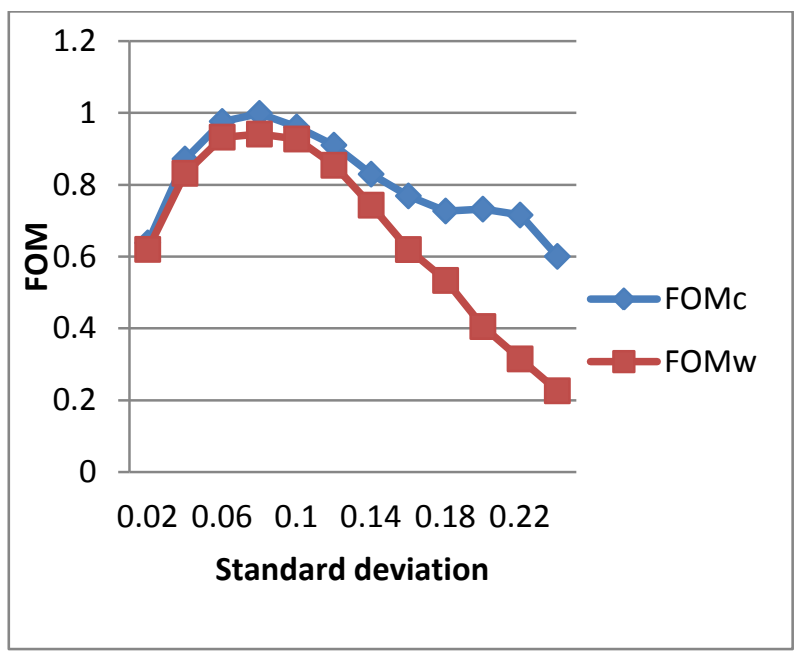

Fig 4: comparison of Contourlet \& Wavelet in the case of FOM.

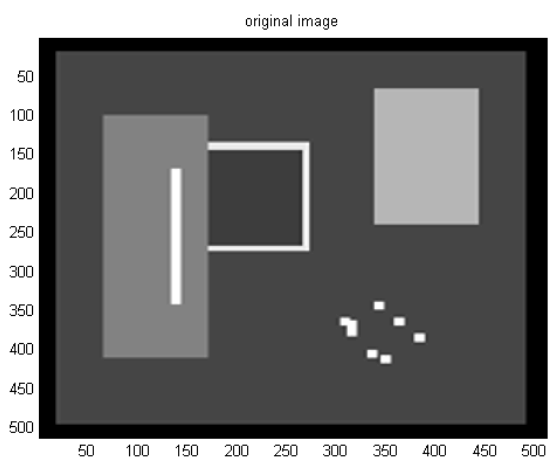

a

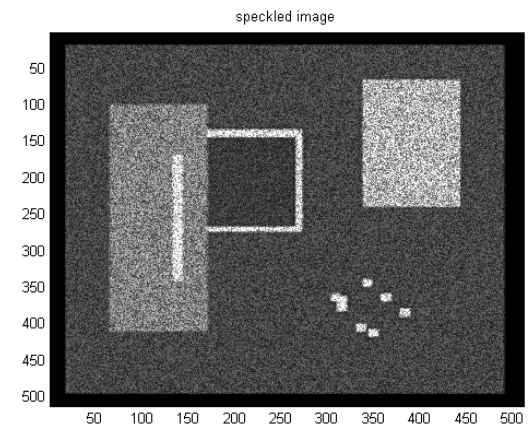

b

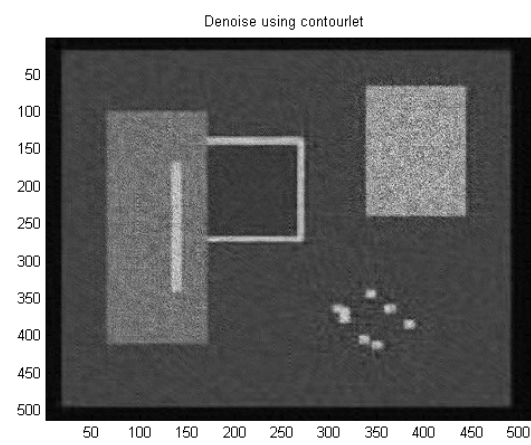

c

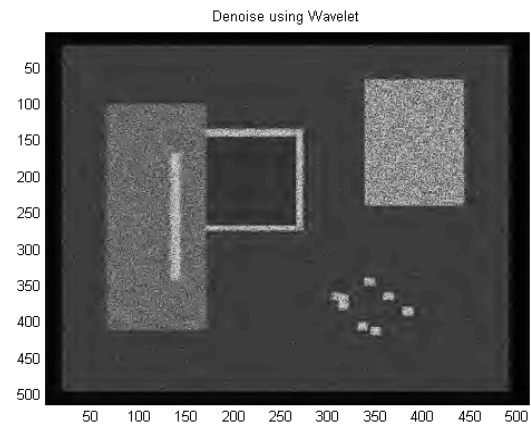

d

Fig 5: (a) Original synthesized image (b) Speckled image (sigma $=0.1)$ (c) Despeckled image with contourlet (d) Despeckled image with wavelet 
Table 1. Results of implementing on synthed image

\begin{tabular}{|c|c|c|c|c|c|c|c|}
\hline$\sigma$ & SNR & SNRw & SNRc & PSNRw & PSNRc & FOMc & FOMw \\
\hline 0.02 & 16.3008 & 17.2864 & 17.5948 & 80.0777 & 79.4523 & 0.6378 & 0.6198 \\
\hline 0.04 & 16.0706 & 17.8604 & 18.4071 & 74.52 & 74.1366 & 0.8713 & 0.8315 \\
\hline 0.06 & 15.8035 & 18.163 & 18.6285 & 71.7584 & 71.3608 & 0.9759 & 0.9322 \\
\hline 0.08 & 15.5586 & 18.6415 & 19.0096 & 69.8971 & 69.5531 & 0.9983 & 0.9407 \\
\hline 0.1 & 15.3819 & 19.161 & 19.3919 & 68.5116 & 68.2912 & 0.9605 & 0.9270 \\
\hline 0.12 & 15.1717 & 19.495 & 19.7172 & 67.4421 & 67.2461 & 0.9099 & 0.8539 \\
\hline 0.14 & 15.0123 & 19.8566 & 19.5963 & 66.5479 & 66.3829 & 0.8291 & 0.7428 \\
\hline 0.16 & 14.841 & 20.439 & 20.1808 & 65.8115 & 65.7193 & 0.7685 & 0.6193 \\
\hline 0.18 & 14.662 & 20.5728 & 19.9319 & 65.1824 & 65.1334 & 0.7266 & 0.5333 \\
\hline 0.2 & 14.5226 & 21.3212 & 19.8752 & 64.6145 & 64.7278 & 0.7318 & 0.4050 \\
\hline 0.22 & 14.3688 & 21.0057 & 19.7785 & 64.1648 & 64.2841 & 0.7154 & 0.3149 \\
\hline 0.24 & 14.1882 & 21.0081 & 19.4151 & 63.7433 & 63.8665 & 0.6002 & 0.2261 \\
\hline
\end{tabular}

\subsection{Compression and speckle reduction results}

To evaluate the performance of the proposed method two common metrics were used for performance assessment of image compression methods. The proposed method was applied on several ultrasound images and the performance of method with was compared with other compression methods. Set partitioning in hierarchical trees (SPIHT) is an image compression algorithm that exploits the inherent similarities across the sub-bands in a wavelet decomposition of an image. This algorithm codes the most important wavelet transform coefficients first, and transmits the bits so that an increasingly refined copy of the original image can be obtained progressively [22]. As SPIHT is one of the accepted compression methods, the results were compared with SPIHT. In order to evaluate the performance of the proposed method it was applied on real ultrasound images and its compression ability and its results are shown in table 3 and figure 7.

Peak Signal to Noise Ratio (PSNR) is one of the quantitative measures for image quality evaluation.

Compression Ratio (CR) is defined as follow:

$\mathrm{CR}=$ Original file size/ Compressed file size

In the case of ultrasound phantom image [26] and for the same compression ratios we compare the PSNR. The results are presented in figure 4 and table 1 . Then we used a speckled fetal ultrasound image [27] to evaluate the speckle reduction and image

Table 2. compression results of ultrasound phantom image

\begin{tabular}{|c|c|c|}
\hline Method & PSNR(db) & CR \\
\hline SPIHT & 25.51 & 4.0313 \\
\hline Proposed method & 79.2566 & 4.1890 \\
\hline
\end{tabular}

\section{CONCLUSION}

In this paper, a new threshold-based method for compression and speckle reduction of medical ultrasound images was presented. In this method, contourlet transform was used and a new algorithm for coding contourlet coefficients was presented. In this procedure, after applying contourlet transform a threshold was selected due to coefficients set. The threshold value is the most frequent contourlet coefficient. So, it is not a predefined value and is defined due to the information content of the image. In this process we can use another threshold for speckle reduction this threshold is chosen due to the variance of the noise. So, our method has the ability of despeckling and compression simultaneously. After thresholding, the coefficients are quantized. Then, Huffman coding is applied on the quantized coefficients. This threshold based lossy compression process reduces the number of coefficients necessary to reconstruct the original image. We compare our method with SPIHT, wavelet based compression method, and the result shows that in the same compression ratio, our method has a better performance in the case of PSNR. The contribution of this paper is selecting threshold value due to information content and simultaneous compression and despeckling. This method can be used in PACS systems and other telemedicine systems due to its god performance and speed. However, it can be developed to reach better performances.

Table 3. compression results of fetal ultrasound image

\begin{tabular}{|c|c|c|}
\hline Method & PSNR(db) & CR \\
\hline SPIHT & 73.32 & 8.223 \\
\hline Proposed method & 79.1702 & 6.692 \\
\hline
\end{tabular}




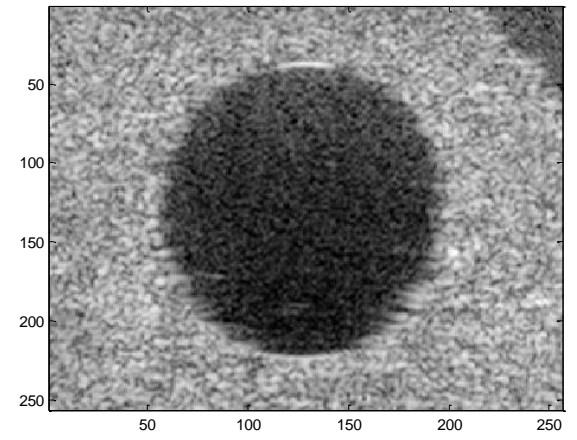

a

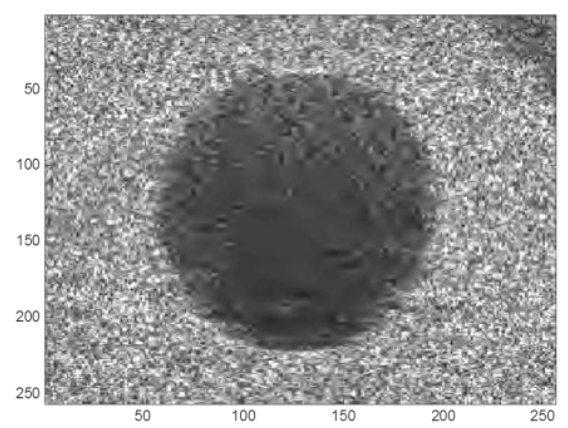

b

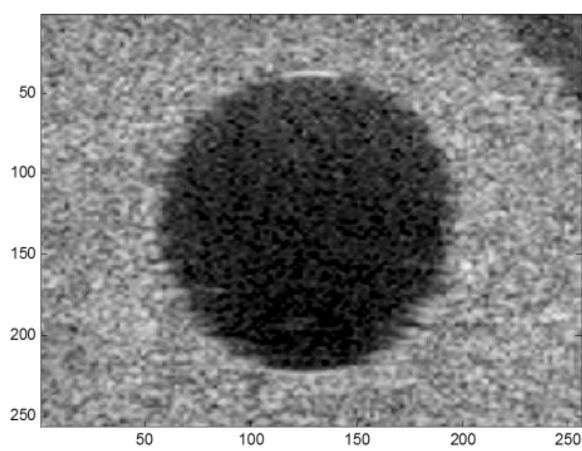

C

Fig 6: Ultrasound phantom image (a) original image

(b) compressed image by SPIHT (c) compressed image with proposed method

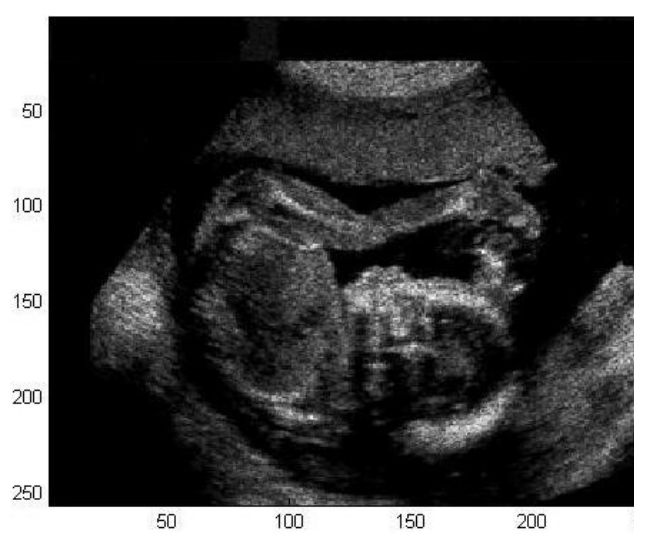

a

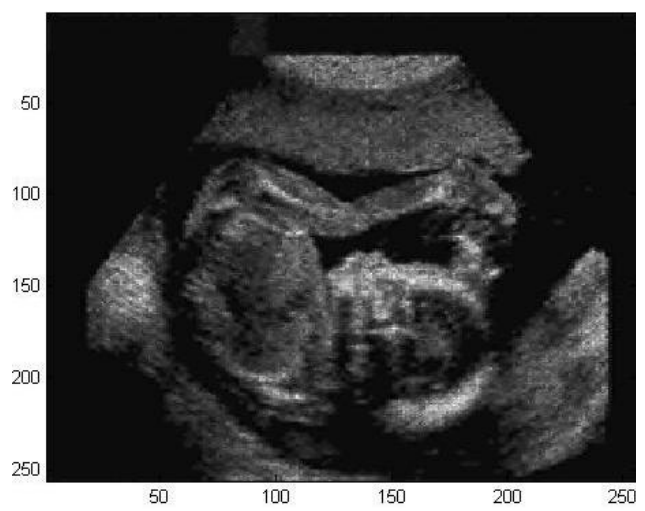

b

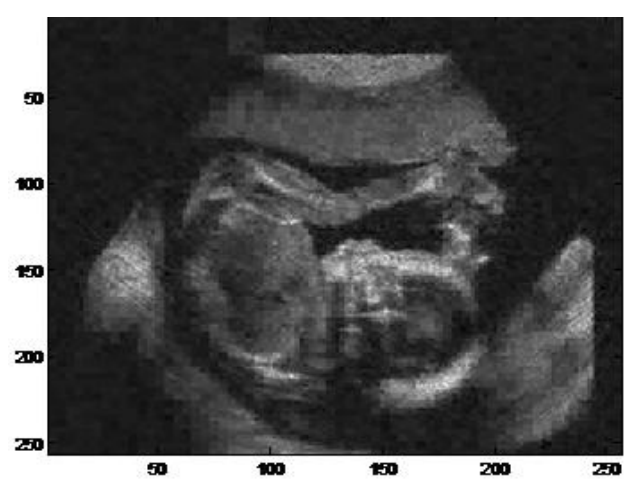

c

Fig 7: (a) original image (b) compressed image with proposed method (c) compressed image by SPIHT 


\section{REFERENCES}

[1] R. F. Wagner, S. W. Smith, J. M. Sandrik, and H. Lopez, 1983. "Statistics of speckle in ultrasound B-scans," IEEE Trans. Ultrasonics., vol. 30, pp. 156-163, (May 1983).

[2] O. V. Michailovich and A. Tannenbaum, 2006. "Despeckling of Medical Ultrasound Images," IEEE Trans. Ultrasonics., vol. 53, no. 1, (Jan. 2006).

[3] J. S. Lee, 1981 "Speckle analysis and smoothing of synthetic aperture radar images," Comput. Graph. Image Processing, vol. 17, pp. 24-32, (1981).

[4] J. S. Lee, 1980 "Digital image enhancement and noise filtering by using local statistics," IEEE Trans. Pattern Anal. Machine Intell., vol. PAMI-2, no. 2, pp. 165-168, (1980)

[5] D. T. Kuan, A. A. Sawchuk, T.C. Strand, and P. chavel, 1987. "Adaptive restoration of images with speckle," IEEE Trans. Acoust. Specch Signal Processing, vol. ASSP-35, pp. 373-383, (1987).

[6] V. S. Frost, J. A. Stiles, K. S. Shanmuggam, and J.C. Holtzman 1982 "Amodel for radar images and its application for adaptive digital filtering of multiplicative noise," IEEE Trans. Pattern Anal. Machine Intell., vol. 4, no. 2, pp. 157-165,(1982).

[7] P. A. Juang and M. N. Wu, 2007. "Ultrasound Speckle Image Process Using Wiener Pseudo-inverse Filtering," in Proc. 2007 The 33rd IEEE Industrial Electronics Society (IECON) Conf., pp. 2446-2449.

[8] T. Huang, G. Yang, and G. Tang, 1979 "A fast twodimensional median filtering algorithm," IEEE Trans. Acoust. Speech Signal Processing, vol. 27, no. 1, pp. 13$18,(1979)$

[9] Lopes, E. Nezry, R. Touzi and H. Laur, 1990. "Maximum A Posteriori speckle filtering and first order texture models in SAR images," in Proc. 10th Geoscience and Remote Sensing Symposium, pp. 24092412, (May 1990)

[10] Y. Yongjian and S. T. Acton, 2002 "Speckle reducing anisotropic diffusion," IEEE Trams. Image Processing, vol.11, no. 11, pp. 1260-1270, (Nov. 2002).

[11] J. S. Lee, 1981 "Refined filtering of image noise using local statistics," Comput. Graph. Image Processing, vol. 15, pp. 380-389, (1981)

[12] S. Zhong and V. Cherkassky, 2000."Image denoising using wavelet thresholding and model selection," in Proc. IEEE Int. Conf. Image Processing, pp. 1-4, Nov. 2000 .
[13] D. L. Donoho, 1995. "Denoising by soft thresholding," IEEE Trans. Inform. Theory, vol.41, pp.613-627, 1995.

[14] F. N. S. Medeiros, N. D. A. Mascarenhas, R. C. P. Marques, and C. M. Larprano, 2002 ."Edge preserving wavelet Speckle filtering," in Proc. 5th IEEE southwest Symp. Image Anal. Interpretation, pp. 281-285, April 7 9, 2002

[15] M. N. Do, and M. Vetterli, 2005. " The Contourlet Transform: An Efficient Directional Multiresolution Image Representation," IEEE Trans. On Image Processing, vol. 14, no. 12, pp. 2091-2106, Dec. 2005.

[16] Parthiban, and R. Subramanian, 2006. " Speckle Noise Removal Using Contourlets," in Proc. International Conference on Information and Automation 2006, pp. 250-253.

[17] K. Jain, 1989. Fundamentals of Digital Image Processing. Englewood Cliffs, NJ: Prentice-Hall, 1989.

[18] R. C. Gonzalez and Richard E. Woods, "Digital Image Processing", Second Edition, Pearson Education.

[19] G. K. Wallace, 1991 "The JPEG still picture compression standard," IEEE Transaction on Consumer Electronics, vol. 38, no. 1, pp. 30-44, (Feb. 1991).

[20] O. Rioul, and M. Vetterli, 1991"Wavelets and Signal Processing," IEEE Transaction on Signal Processing, vol. 8, no. 4, pp. 14-38. (1991).

[21] M. Shapiro, 1993. "Embedded Image Coding Using Zero trees of Wavelet Coefficients," IEEE Transaction on Signal Processing, vol. 41, no. 12, pp. 3445-3462, (1993).

[22] Said, and W. A. Pearlman, 1996“Anew Fast and Efficient Image Codec Based on Set Partitioning in Hierarchical Trees," IEEE Transaction on Circuits and Systems for Video Technology, vol. 6, no. 3, pp. 243250, (1996)

[23] D. S. Taubman, 2000"High performance scalable image compression with EBCOT," IEEE Transaction on Image Processing, vol. 9, no. 7, pp. 1158-1170, (2000)

[24] Y. Fisher, 1995 "Fractal Image compression: Theory and Application”, Springer Verlag, New York, (1995).

[25] M. N. Do, and M. Vetterli, 2005. "The Contourlet Transform: An Efficient Directional Multiresolution Image Representation," IEEE Trans. On Image Processing, vol. 14, no. 12, pp. 2091-2106, Dec.2005.

[26] http://viva.ee.virginia.edu/downloads.html

[27] http://www.ee.nmt.edu/ erives/552_11/EE552.html 